\title{
Administration of the selective cyclooxygenase (COX)-2 inhibitor etodolac prolongs cardiac allograft survival in a mouse model
}

\author{
MASAHIDE MATSUYAMA $^{1}$, RIKIO YOSHIMURA ${ }^{2}$, TARO HASE ${ }^{2}$, JAMEL CHARGUI $^{1}$, \\ NORIO YOSHIMURA ${ }^{3}$ and JEAN-LOUIS TOURAINE ${ }^{1}$
}

\begin{abstract}
${ }^{1}$ Department of Transplantation and Clinical Immunology, Claude Bernard University of Lyon and Lyon Hospitals, Lyon, France;
${ }^{2}$ Department of Urology, Osaka City University Graduate School of Medicine, Osaka; ${ }^{3}$ Department of Transplantation and Regenerative Surgery, Graduate School of Medical Science, Kyoto Prefectural University of Medicine, Kyoto, Japan
\end{abstract}

Received March 8, 2010; Accepted July 21, 2010

DOI: $10.3892 / \mathrm{mmr} .2010 .339$

\begin{abstract}
Etodolac, a selective cyclooxygenase-2 (COX-2) inhibitor, is a non-steroidal anti-inflammatory drug. COX-2 is a key factor in the progression of inflammation. Although inflammation is an essential pathologic feature of cardiac allograft rejection, the role of COX-2 in this process remains unclear. The aim of this study was to investigate the expression of COX and the effects of etodolac in a mouse cardiac allograft model. Balb/c mice $(\mathrm{H}-2 \mathrm{~d})$ were used as recipients and $\mathrm{C} 57 \mathrm{BL} / 6(\mathrm{H}-2 \mathrm{~b})$ mice as heart donors. Heart function was evaluated daily after transplantation by regular abdominal palpation of the heart and by laparotomy in cases where the beating became weak. Rejection was defined as total cessation of cardiac muscle contraction. COX-2 expression was analyzed by immunohistochemistry. Cardiac isograft was well tolerated ( $>150$ days, $n=5$ ), while non-treated cardiac allograft was rapidly rejected (mean $10.9 \pm 2.4, n=7$ ). In the etodolactreated cardiac allograft $(10 \mathrm{mg} / \mathrm{kg} /$ day by hypodermic injection), survival was extended to $18.53 \pm 2.1$ days $(n=7)$. The necrotic area and the grade of COX-2 immunostaining were more significantly reduced in the etodolac-treated cardiac allograft than in the non-treated cardiac allograft at day 14 These results indicate that etodolac contributes to protection against rejection after heart transplantation. Etodolac could therefore be used to suppress graft rejection by means of its anti-inflammatory properties.
\end{abstract}

\section{Introduction}

Myocardial inflammation constitutes a major component of the pathologic changes observed during cardiac allograft

Correspondence to: Dr Masahide Matsuyama, Department of Transplantation and Regenerative Surgery, Graduate School of Medical Science, Kyoto Prefectural University of Medicine, 465 Kajiicho, Kawaramachi, Kamigyou-ku, Kyoto 602-0841, Japan E-mail: jasmin@koto.kpu-m.ac.jp

Key words: cyclooxygenase-2, etodolac, mice cardiac allograft, survival rejection. Prostaglandins (PGs), along with leukotrienes and lipoxins, are lipid mediators which contribute to the vasodialation, edema and plasma protein leakage that occur during the inflammatory response (1).

The metabolism of arachidonic acid by the cyclooxygenase $(\mathrm{COX})$ pathway generates eicosanoids, which have been implicated in the pathogenesis of a variety of human diseases, including heart disease. COX is the first enzyme in the pathway responsible for producing PGs and thromboxane from arachidonic acid. There are two isoforms of COX, COX-1 and COX-2. COX-1 occurs in tissues and cells and works to protect the cell. COX-2 is expressed momentarily and strongly on growth factors, promotors and certain endotoxins, and is involved in inflammation, cell growth and differentiation. COX-1 is constitutively expressed in most tissues, whereas COX-2 is induced in response to pro-inflammatory cytokines and stress (2).

It is believed that COX-2 is induced during both acute and chronic inflammatory responses, and is primarily responsible for the PG synthesis that ensues. This has led to the development of drugs that selectively inhibit PG production by COX-2, thus preventing adverse consequences, such as gastric ulcers, that may result from the inhibition of COX-1 (3). The PGs in particular play a role in the pathogenesis of inflammation involving cell-mediated immune responses, such as those that occur in rheumatoid arthritis and allograft rejection.

In a cardiac allograft model, it has been reported that COX-2 mRNA and enzyme proteins are up-regulated during cardiac allograft rejection (1). Therefore, the present study was designed to investigate whether COX-2 is expressed in a mouse cardiac allograft model and whether the selective COX-2 inhibitor etodolac helps inhibit myocardial inflammation during cardiac allograft rejection.

\section{Materials and methods}

Animals. Balb/c (H-2d) and C57BL/6 (H-2b) mice were obtained from the original animal facilities at Claude Bernard University, Edouard Herriot Hospital.

Heart transplantation. Surgical procedures were performed under anesthesia by intra-peritoneal injection of Hypnomidate 

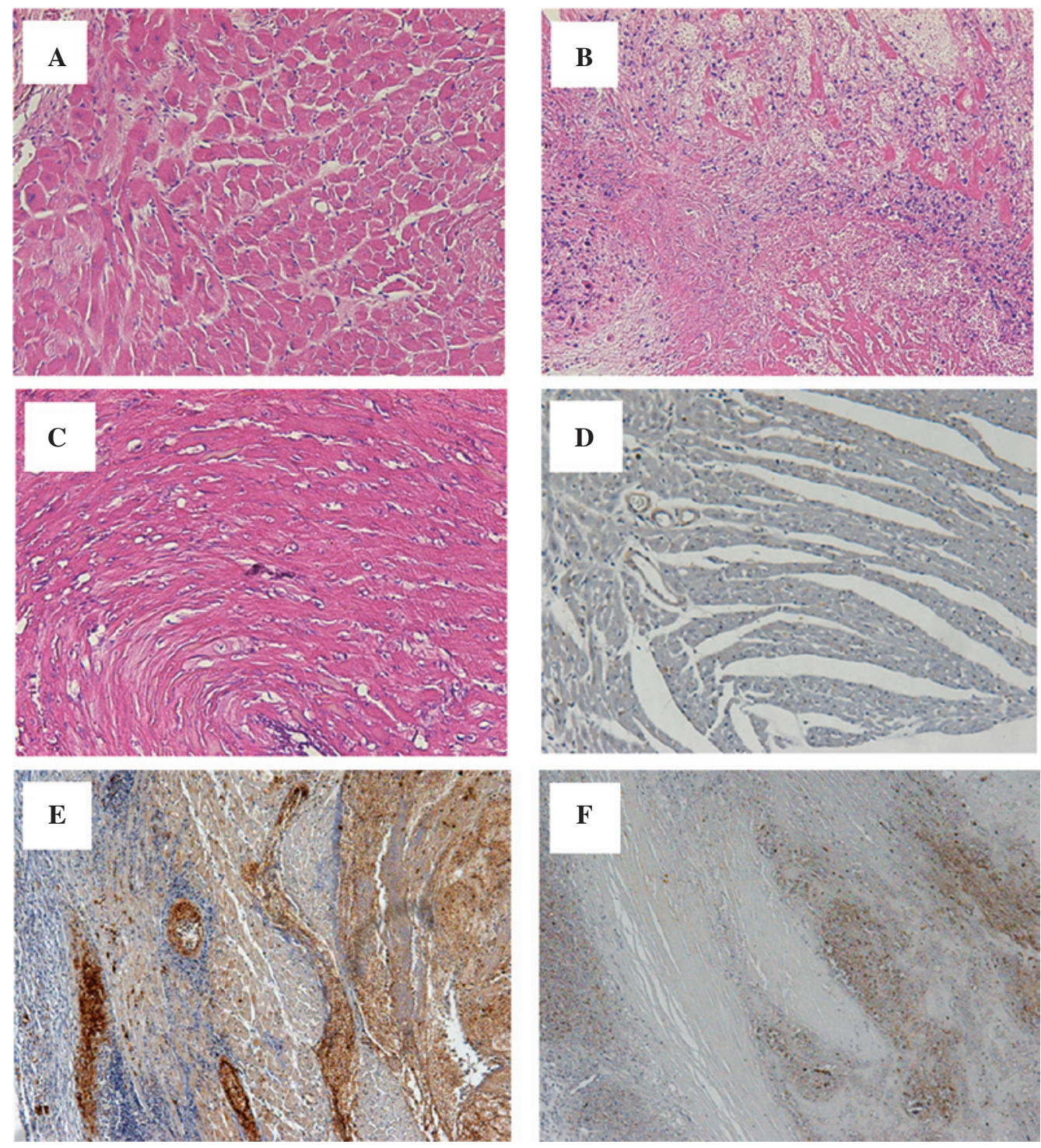

Figure 1. H\&E staining (A-C) and COX-2 immunohistochemistry (D-F) from samples collected on day 14. (A) H\&E staining of the cardiac isograft revealed normal tissue architecture, with no damaged myocardia. (B) In the non-treated cardiac allograft, extensive lymphocyte infiltration was observed, and almost all the myocardia were damaged and destroyed. Complete rejection occurred. (C) In the etodolac-treated cardiac allograft, some areas were infiltrated by lymphocytes and some myocardia were damaged and destroyed. However, the majority of myocardia were preserved. (D) In the cardiac isograft, slight COX-2 immunostaining was observed in endothelial cells. (E) In the non-treated cardiac allograft, marked COX-2 immunostaining was observed in macrophages, damaged cardiomyocytes and endothelial cells, and inflammatory infiltrate. (F) In the etodolac-treated cardiac allograft, COX-2 immunostaining was most apparent in the infiltrating macrophages, with decreased immunostaining of cardiac myocytes.

$(20 \mathrm{mg} / \mathrm{kg})$ and Midazoram $(5 \mathrm{mg} / \mathrm{kg})$. Vascularized heterotopic heart grafts from $\mathrm{B} 6(\mathrm{H}-2 \mathrm{~b})$ mice were placed in the abdomen of $\mathrm{BALB} / \mathrm{c}(\mathrm{H}-2 \mathrm{~d})$ recipient mice and anastomosed to the abdominal aorta and vena cava, as we described previously (4). Heart function was evaluated daily after transplantation by regular abdominal palpation of the transplanted heart and by laparotomy in cases where the beating became weak. Rejection was defined as total cessation of cardiac muscle contraction.

Selective COX-2 inhibitor administration. The selective COX-2 inhibitor etodolac was obtained from Nippon Shinyaku Co., Ltd. (Kyoto, Japan). Recipient mice were injected subcutaneously with etodolac $(10 \mathrm{mg} / \mathrm{kg} /$ day by hypodermic injection) daily from day 0 as the treatment group. Control group mice were treated with the same volume of phosphatebuffered saline (PBS). Etodolac has anti-inflammatory effects with a better safety profile for the stomach than non-selective non-steroidal anti-inflammatory drugs (NSAIDs) (5).

Histology and immunohistochemistry. Cardiac allograft tissue specimens were collected on day 14 and preserved in $10 \%$ formalin, embedded in paraffin, serially sectioned onto microscope slides at a thickness of $4 \mu \mathrm{m}$, and finally deparaffinized. For routine histologic examination, sections were stained with H\&E to determine the extent and severity of rejection according to the International Society of Heart and Lung Transplantation classification (6). Immunohistochemical staining was performed with the VectaStain avidin-biotin peroxidase complex kit (Vector Laboratories, CA, USA) as previously described (7). Primary polyclonal antibodies against mouse COX-2 (1:50 dilution in PBS) (Cayman Chemical, MI, USA) and control PBS were used. 


\section{Results}

Allograft survival. Cardiac isograft was well tolerated $(>150$ days, $n=5)$, while non-treated cardiac allograft was rapidly rejected (mean $10.9 \pm 2.4, \mathrm{n}=7$ ). In the etodolac-treated cardiac allograft $(10 \mathrm{mg} / \mathrm{kg} /$ day by hypodermic injection), survival was extended to $18.53 \pm 2.1$ days $(n=7)$.

$H \& E$ staining. $H \& E$ staining of the cardiac isograft tissues revealed normal tissue architecture, with no damaged myocardia (Fig. 1A). In the non-treated cardiac allograft, extensive lymphocyte infiltration was observed and almost all the myocardia were damaged and destroyed. Complete rejection occurred (Fig. 1B). In the etodolac-treated cardiac allograft, some areas were infiltrated by lymphocytes and some myocardia were damaged and destroyed. However, the majority of myocardia were preserved (Fig. 1C).

Immunohistochemistry. In the cardiac isograft tissues, slight COX-2 immunostaining was observed in endothelial cells (Fig. 1D). In the non-treated cardiac allograft, marked COX-2 immunostaining was observed in macrophages, damaged cardiomyocytes and endothelial cells, and inflammatory infiltrate (Fig. 1E). In the etodolac-treated cardiac allograft, COX-2 immunostaining was most apparent in the infiltrating macrophages, with decreased immunostaining of cardiac myocytes (Fig. 1F).

\section{Discussion}

Myocardium inflammation (leukocyte infiltration and interstitial edema) along with contractile dysfunction and death of cardiac muscle cells are hallmarks of acute cardiac allograft rejection $(8,9)$. Along with cytokines, histamine and kinins, the eicosanoids are a family of lipid mediators that contribute to the vasodialation and leakage of plasma proteins and fluid into the interstitial space, which occurs during the acute inflammatory response in the myocardium and in other tissues (10).

COX, which produces prostanoids from arachidonic acid, is the principal target of NSAIDs. Recently, two COX isoforms, COX-1 and COX-2, were identified. Both the COX-1 and COX-2 enzymes are transformed from cell membrane phospholipids to arachidonic acid by phospholipase $\mathrm{A}_{2}$, and then transform arachidonic acid to $\mathrm{PGH}_{2}$ through $\mathrm{PGG}_{2}$. COX-1 is present in most tissues and is involved in the physiological production of PGs for the maintenance of normal homeostasis. COX-2 is well-established to participate in prostanoid production and is implicated in the processes of disease and expressed in inflammatory sites that often have harmful effects. COX-2 expression is therefore associated with pain, fever, lipopolysaccharide stimulation, ischemia/reperfusion injury and allograft rejection (11).

In cardiovascular diseases, COX-2 is known to have a cardioprotective protection effect that alleviates ischemia/ reperfusion injury, mediating the late phase of pre-conditioning (12). Since acute cardiac rejection of transplanted heart is known to cause serious myocardial inflammation, COX-2 is enhanced in rejected cardiac allografts (1). The inhibition of COX-2 prolongs cardiac allograft survival and reduces myocardial damage and inflammation during acute cardiac allograft rejection in a rat model (10).

Several studies corroborate our data. Yang et al reported that COX-2 expression is enhanced in the myocardium during cardiac allograft rejection by immunohistochemistry in a rat model (1). Ningsheng et al also reported that the administration of the COX-2 inhibitor DFU ( $5 \mathrm{mg} / \mathrm{kg}$ /day by intraperitoneal injection) prolongs cardiac allograft survival (from $6.3 \pm 0.5$ to $12.6 \pm 2.6$ days, $\mathrm{P}=0.01$ ) and reduces myocardial damage and inflammation during acute cardiac allograft rejection in a Lewis rat model (10). In a mouse model, Ogawa et al reported that the COX-2 inhibitor meloxicam $(0.1 \mathrm{mg} /$ $\mathrm{kg} /$ day intraperitoneal injection) suppressed inflammation and fibrosis in cardiac allografts. However, they found that non-treated cardiac allografts were acutely rejected $(7.8 \pm 0.4$ days, $n=6)$ and that COX-2 inhibitor did not prolong cardiac allograft survival (7.5 \pm 4.2 days, $n=6)(13)$.

It is well known that meloxicam and etodolac are selective COX-2 inhibitors. There are therefore several possible reasons for the differing results of the abovementioned studies. First, etodolac has higher selectivity than meloxicam $(14,15)$. Second, etodolac is not associated with a statistically increased risk of acute myocardial infarction compared to other COX-2 inhibitors (16). Etodolac has been associated with cardionegative or cardioprotective effects in studies comparing them to NSAIDs (17). Finally, etodolac showed the highest UD(50) value and safety index as compared to NSAIDs and meloxicam in arthritic rats (5). These differences may be responsible for the prolongation of cardiac allograft survival by etodolac.

In conclusion, the administration of etodolac prolongs cardiac allograft survival and reduces myocardial damage and inflammation during acute cardiac allograft rejection.

The present study demonstrates that the selective COX-2 inhibitor etodolac, administered by subcutaneous injection, prolongs cardiac allograft survival and reduces myocardial damage and inflammation during cardiac allograft rejection in a mouse model. These results suggest that, with further study, etodolac may emerge as another agent in the therapeutic armamentarium for the treatment of patients undergoing heart transplantation.

\section{References}

1. Yang X, Ma N, Szabolcs MJ, et al: Upregulation of COX-2 during cardiac allograft rejection. Circulation 101: 430-438, 2000.

2. Matsuyama M and Yoshimura R: Relationship between cyclooxygenase (COX)-2 and malignant tumors. Transworld Research Network. Recent Res Devel Cancer 6: 243-251, 2004.

3. Masferrer JL, Zweifel BS, Manning PT, et al: Selective inhibition of inducible cyclooxygenase-2 in vivo is anti-inflammatory and nonulcerogenic. Proc Natl Acad Sci USA 91: 3228-3232, 1994.

4. Hase T, Chargui J, Inori F, et al: Human interleukin-10 transduced fetal liver stem cells prolong survival of mouse skin and heart allografts. Transplant Proc 37: 287-288, 2005.

5. Tachibana M, Inoue N, Yoshida E, Matsui M, Ukai Y and Yano J: Anti-inflammatory effect and low ulcerogenic activity of etodolac, a cyclooxygenase- 2 selective non-steroidal anti-inflammatory drug, on adjuvant-induced arthritis in rats. Pharmacology 68: 96-104, 2003

6. Billingham ME, Cary NR, Hammond ME, et al: A working formulation for the standardization of nomenclature in the diagnosis of heart and lung rejection: Heart Rejection Study Group. The International Society for Heart Transplantation. J Heart Transplant 9: 587-593, 1990 
7. Sano H, Hla T, Maier JA, et al: In vivo cyclooxygenase expression in synovial tissues of patients with rheumatoid arthritis and osteoarthritis and rats with adjuvant and streptococcal cell wall arthritis. J Clin Invest 89: 97-108, 1992.

8. Yang X, Chowdhury N, Cai B, et al: Induction of myocardial nitric oxide synthase by cardiac allograft rejection. J Clin Invest 94: 714-721, 1994.

9. Worrall NK, Lazenby WD, Misko TP, et al: Modulation of in vivo alloreactivity by inhibition of inducible nitric oxide synthase. J Exp Med 181: 63-70, 1995.

10. Ma N, Szabolcs MJ, Sun J, et al: The effect of selective inhibition of cyclooxygenase (COX)-2 on acute cardiac allograft rejection. Transplantation 74: 1528-1534, 2002.

11. Simon LS: Role and regulation of cyclooxygenase-2 during inflammation. Am J Med 106: 37-42, 1999.

12. Bolli R, Shinmura K, Tang XL, et al: Discovery of a new function of cyclooxygenase (COX)-2: COX-2 is a cardioprotective protein that alleviates ischemia/reperfusion injury and mediates the late phase of preconditioning. Cardiovasc Res 55: 506-519, 2002.

13. Ogawa M, Suzuki J, Koga N, Kosuge H and Isobe M: A specific COX-2 inhibitor attenuates cell infiltration but does not prolong graft survival in murine cardiac transplantation. Transplant Proc 37: 121-122, 2005
14. Warner TD, Giuliano F, Vojnovic I, Bukasa A, Mitchell JA and Vane JR: Nonsteroid drug selectivities for cyclo-oxygenase-1 rather than cyclo-oxygenase-2 are associated with human gastrointestinal toxicity: a full in vitro analysis. Proc Natl Acad Sci USA 96: 7563-7568, 1999.

15. Kato M, Nishida S, Kitasato H, Sakata N and Kawai S: Cyclooxygenase-1 and cyclooxygenase- 2 selectivity of nonsteroidal anti-inflammatory drugs: investigation using human peripheral monocytes. J Pharm 53: 1679-1685, 2001.

16. Warner JJ, Weideman RA, Kelly KC, et al: The risk of acute myocardial infarction with etodolac is not increased compared to naproxen: a historical cohort analysis of a generic COX-2 selective inhibitor. J Cardiovasc Pharmacol Ther 13: 252-260, 2008.

17. Motsko SP, Rascati KL, Busti AJ, et al: Temporal relationship between use of NSAIDs, including selective COX-2 inhibitors, and cardiovascular risk. Drug Saf 29: 621-632, 2006. 